\title{
A UNIFIED PROOF FOR THE CONVERGENCE OF JACOBI AND GAUSS-SEIDEL METHODS *
}

\author{
ROBERTO BAGNARA ${ }^{\dagger}$
}

\begin{abstract}
We present a new unified proof for the convergence of both the Jacobi and the Gauss-Seidel methods for solving systems of linear equations under the criterion of either (a) strict diagonal dominance of the matrix, or (b) diagonal dominance and irreducibility of the matrix. These results are well known. The proof for criterion (a) makes use of Geršgorin's theorem, while the proof for criterion (b) uses Taussky's theorem that extends Geršgorin's work. Hence the topic is interesting for teaching purposes.
\end{abstract}

Key words. Systems of equations, iterative methods.

AMS subject classifications. $65-01,65 \mathrm{~F} 10$

We present a unified proof for the convergence of both the Jacobi and the GaussSeidel iterative methods for solving systems of linear equations under the criterion of either (a) strict diagonal dominance of the matrix, or (b) diagonal dominance and irreducibility of the matrix. These results are well known [2]. For each criterion, the proof is unified in that it uses similar arguments for both the methods. The proof is simple because low level details are contained in an easy Lemma and a couple of Observations, while the rest of the analysis is based on elementary properties of matrices and their eigenvalues.

We recall three fundamental results about the location of the eigenvalues, known as Geř̌gorin type theorems [1]. Let $A \in \mathbf{C}^{n \times n}$ be a complex matrix: the Geřsgorin (row) disks of $A$ are given, for $i=1, \ldots, n$, by

$$
K_{i}=\left\{z \in \mathbf{C}|| z-a_{i i}\left|\leq \sum_{\substack{j=1 \\ j \neq i}}^{n}\right| a_{i j} \mid\right\}
$$

The first Geršgorin theorem says that the eigenvalues of $A$ are contained within the union, $K$, of the disks, where

$$
K=\bigcup_{i=1}^{n} K_{i}
$$

The second Geršgorin theorem says further that any union of $k$ disks of $A$ not intersecting the remaining $(n-k)$ disks, must contain exactly $k$ of the eigenvalues (counting multiplicities).

The third Geršgorin type theorem was established by Taussky [1] and says that if $A$ is irreducible and has an eigenvalue $\lambda$ on the boundary of $K$, then $\lambda$ is on the boundary of each of the $n$ Geršgorin disks of $A$. Notice that since $A$ and $A^{\mathrm{T}}$ have exactly the same eigenvalues, analogous results hold when column sums of $A$, instead of row sums, are used to define the radii of the corresponding (column) disks.

\footnotetext{
*Received by the editors July 21, 1993; accepted for publication (in revised form) July 14, 1994.

†Dipartimento di Informatica, Università di Pisa, Corso Italia, 40, 56125 Pisa, Italy (bagnara@di.unipi.it).
} 
We now define the terms used in criteria (a) and (b):

Definition 1. A complex matrix $A \in \mathbf{C}^{n \times n}$ is reducible iff there exist a permutation matrix $\Pi$ (i.e., $\Pi$ is obtained from the identity, $I$, by a permutation of the rows of $I)$ and an integer $k \in\{1, \ldots, n-1\}$ such that

$$
\Pi A \Pi^{\mathrm{T}}=\left(\begin{array}{cc}
A_{11} & A_{12} \\
\mathbf{0} & A_{22}
\end{array}\right)
$$

where $A_{11}$ is $k \times k$ and $A_{22}$ is $(n-k) \times(n-k)$. If $A$ is not reducible, than $A$ is said to be irreducible (see [1] for further discussion of irreducibility).

Note that premultiplication of $A$ by $\Pi$, produces a matrix $\Pi A$ that consists of the rows of $A$ in the same order that the rows of $I$ appear in $\Pi$. On the other hand, when the columns of $I$ are permuted in that order, then the matrix $\Pi^{\mathrm{T}}$ is produced. Thus, $A \Pi^{\mathrm{T}}$ is a matrix consisting of the columns of $A$ in that same order.

Definition 2. $A \in \mathbf{C}^{n \times n}$ is said to be strictly diagonally dominant (by rows) iff, for each $i=1, \ldots, n$,

$$
\left|a_{i i}\right|>\sum_{\substack{j=1 \\ j \neq i}}^{n}\left|a_{i j}\right|
$$

similarly, $A$ is said to be strictly diagonally dominant (by columns) iff, for each $j=1, \ldots, n$,

$$
\left|a_{j j}\right|>\sum_{\substack{i=1 \\ i \neq j}}^{n}\left|a_{i j}\right|
$$

Definition 3. $A \in \mathbf{C}^{n \times n}$ is said to be diagonally dominant (by rows) iff, for each $i=1, \ldots, n$,

$$
\left|a_{i i}\right| \geq \sum_{\substack{j=1 \\ j \neq i}}^{n}\left|a_{i j}\right| \quad \text { and } \exists s \in\{1, \ldots, n\} \text { such that } \quad\left|a_{s s}\right|>\sum_{\substack{j=1 \\ j \neq s}}^{n}\left|a_{s j}\right|
$$

similarly, $A$ is said to be diagonally dominant (by columns) iff, for each $j=1, \ldots, n$,

$$
\left|a_{j j}\right| \geq \sum_{\substack{i=1 \\ i \neq j}}^{n}\left|a_{i j}\right| \quad \text { and } \exists t \in\{1, \ldots, n\} \text { such that } \quad\left|a_{t t}\right|>\sum_{\substack{i=1 \\ i \neq t}}^{n}\left|a_{i t}\right| .
$$

Here are a couple of observations which are useful in proving the convergence theorem:

OBSERvation 1. If the matrix, $A$, is strictly diagonally dominant, then $A$ is nonsingular.

Proof. None of the Geršgorin disks of $A$ contain the origin and therefore, by the first Geršgorin theorem, zero is not an eigenvalue of $A$.

OBSERVATION 2. If the matrix, A, is irreducible and diagonally dominant, then $A$ is nonsingular.

Proof. Otherwise, if $A$ were singular, then one of its eigenvalues would be zero and, by the first Geršgorin theorem, would be contained in at least one of the disks. 
But, by the assumed diagonal dominance, zero cannot be in the interior of any of the disks. Thus, zero would have to be on the boundary of the union, $K$, of the disks. However, Taussky's theorem would then place zero on the boundary of each of the disks. But, this would be impossible for either of the disks labelled $s$ or $t$ in Definition 3.

The Jacobi and Gauss-Seidel iterative methods to solve the system

$$
A \mathbf{x}=\mathbf{b}
$$

where $A \in \mathbf{C}^{n \times n}, \mathbf{x}, \mathbf{b} \in \mathbf{C}^{n}$, are formulated in terms of a decomposition of the matrix A:

$$
A=D-B-C
$$

where the components of $D, B$, and $C$ are defined by

(10) $d_{i j}=\left\{\begin{array}{ll}a_{i j}, & \text { if } i=j, \\ 0, & \text { if } i \neq j ;\end{array} \quad b_{i j}=\left\{\begin{array}{ll}-a_{i j}, & \text { if } i>j, \\ 0, & \text { if } i \leq j ;\end{array} \quad c_{i j}= \begin{cases}-a_{i j}, & \text { if } i<j, \\ 0, & \text { if } i \geq j .\end{cases}\right.\right.$

Both iterative methods begin with an arbitrary initial vector $\mathbf{x}^{0} \in \mathbf{C}^{n}$ and then produce a sequence of vectors, $\mathbf{x}^{k}$, for $k=1,2,3, \ldots$, by solving either:
(11) JACOBI:
$D \mathbf{x}^{k+1}=(B+C) \mathbf{x}^{k}+\mathbf{b}, \quad$ or
(12) Gauss-SEIDEL:$$
(D-B) \mathbf{x}^{k+1}=C \mathbf{x}^{k}+\mathbf{b},
$$

for $k=0,1,2, \ldots$. It is clear that, for the Jacobi method to be applicable, $D$ must be invertible. Similarly, the invertibility of $(D-B)$ is required in order to apply the Gauss-Seidel method. The iteration matrices of the methods are then given, respectively, by
(13) JACOBI:
$J=D^{-1}(B+C)$,
(14) Gauss-SeIdeL:
$G=(D-B)^{-1} C$.

The iterative steps of the methods are then defined, respectively, by
(15) JACOBI:
$\mathbf{x}^{k+1}=J \mathbf{x}^{k}+D^{-1} \mathbf{b}, \quad$ or
(16) Gauss-SEIDEL:

$$
\mathbf{x}^{k+1}=G \mathbf{x}^{k}+(D-B)^{-1} \mathbf{b},
$$

for $k=0,1,2, \ldots$ Let $\rho[Q]$ denote the spectral radius of the matrix $Q$, for any $Q \in \mathbf{C}^{n \times n}$. The Jacobi method is convergent iff $\rho[J]<1$, while the Gauss-Seidel method is convergent iff $\rho[G]<1$.

Theorem 1 (convergence). Let $A \in \mathbf{C}^{n \times n}$ be decomposed as in (9), and let $J$ and $G$ be defined as in (13) and (14), respectively. Under either

the criterion (a): A is strictly diagonally dominant; or

the criterion (b): $A$ is diagonally dominant and irreducible, then

(i) both $D$ and $(D-B)$ are invertible; and

(ii) $\rho[J]<1$, and $\rho[G]<1$.

Proof of (i). For criterion (a). We see that $a_{i i} \neq 0$ for all $i=1, \ldots, n$, whence $D$ and $(D-B)$ are nonsingular.

For criterion (b). We note by Observation 2 that $A$ is nonsingular and hence does not have a zero row. On the other hand, if either $D$ or $(D-B)$ were assumed to be singular, then $d_{k k}=0$ for some $k=1, \ldots, n$. But, then by diagonal dominance we would see that row $k$ of $A$ would be a zero row. This contradiction establishes (i) for criterion (b). 
Proof of (ii). In order to show that both spectral radii are less than 1 , we define the matrices $A_{J}(\lambda)$ and $A_{G}(\lambda)$, where $\lambda \in \mathbf{C}$, by

$$
\begin{aligned}
& A_{J}(\lambda)=\lambda D-B-C ; \\
& A_{G}(\lambda)=\lambda(D-B)-C ;
\end{aligned}
$$

and establish the following:

Lemma. For each $\lambda \in \mathbf{C}$, with $|\lambda| \geq 1$, if A satisfies any of the following properties:

(a) A is strictly diagonally dominant (by rows or by columns);

(b) A is diagonally dominant (by rows, or by columns);

(c) $A$ is irreducible;

then both $A_{J}(\lambda)$ and $A_{G}(\lambda)$ satisfy the same properties.

Proof. (a) Let $A$ be strictly diagonally dominant by rows (the proof for the other case is almost the same). By hypothesis, for each $i=1, \ldots, n$,

$$
\left|a_{i i}\right|>\sum_{\substack{j=1 \\ j \neq i}}^{n}\left|a_{i j}\right|,
$$

if $|\lambda| \geq 1$ then, for each $i=1, \ldots, n$,

$$
\begin{aligned}
\left|\lambda a_{i i}\right| & =|\lambda|\left|a_{i i}\right| \\
& >|\lambda| \sum_{\substack{j=1 \\
j \neq i}}^{n}\left|a_{i j}\right| \\
& =|\lambda| \sum_{j=1}^{i-1}\left|a_{i j}\right|+|\lambda| \sum_{j=i+1}^{n}\left|a_{i j}\right| \\
\geq & |\lambda| \sum_{j=1}^{i-1}\left|a_{i j}\right|+\sum_{j=i+1}^{n}\left|a_{i j}\right| \\
& =\sum_{j=1}^{i-1}\left|\lambda a_{i j}\right|+\sum_{j=i+1}^{n}\left|a_{i j}\right| \\
\geq & \sum_{j=1}^{i-1}\left|a_{i j}\right|+\sum_{j=i+1}^{n}\left|a_{i j}\right| \quad \text { hence the thesis for } A_{G}(\lambda),
\end{aligned}
$$

(b) Very similar to (a). The only difference is that the hypothesis ensures that strict inequality holds in the disequation marked with $(*)$ for at least one $i \in\{1, \ldots, n\}$. Weak inequality is guaranteed for all the other cases.

(c) Since the three matrices $A, A_{J}(\lambda)$, and $A_{G}(\lambda)$, for $\lambda \neq 0$, have zero components in exactly the same locations, it follows that if a permutation matrix $\Pi$ reduces one of these matrices, then $\Pi$ also reduces the other two matrices.

We now resume the proof of (ii), Theorem 1 . The eigenvalues $\lambda$ of $J$ are all and only the solutions of the equation

$$
\operatorname{det}(\lambda I-J)=0
$$


but, from properties of matrices and determinants we have

$$
\begin{aligned}
\operatorname{det}(\lambda I-J) & =\operatorname{det}\left(\lambda I-D^{-1}(B+C)\right) \\
& =\operatorname{det}\left(\lambda D^{-1} D-D^{-1}(B+C)\right) \\
& =\operatorname{det}\left(D^{-1}(\lambda D-B-C)\right) \\
& =\operatorname{det}\left(D^{-1}\right) \operatorname{det}(\lambda D-B-C) \\
& =\operatorname{det}\left(D^{-1}\right) \operatorname{det}\left(A_{J}(\lambda) .\right.
\end{aligned}
$$

So, for (21) to hold we must have $\operatorname{det}\left(A_{J}(\lambda)=0\right.$, as we have already shown that $D$ is nonsingular. But, since $A_{J}(\lambda)$ is nonsingular for $|\lambda| \geq 1$ (by the Lemma and the Observations), it follows that all of the eigenvalues of $J$ are in the interior of the unit circle. Hence, $\rho[J]<1$.

Similarly, the eigenvalues $\lambda$ of $G$ are all and only the solutions of the equation

$$
\begin{aligned}
\operatorname{det}(\lambda I-G) & =\operatorname{det}\left(\lambda I-(D-B)^{-1} C\right) \\
& =\operatorname{det}\left(\lambda(D-B)^{-1}(D-B)-(D-B)^{-1} C\right) \\
& =\operatorname{det}\left((D-B)^{-1}(\lambda(D-B)-C)\right) \\
& =\operatorname{det}\left((D-B)^{-1}\right) \operatorname{det}(\lambda(D-B)-C) \\
& =\operatorname{det}\left((D-B)^{-1}\right) \operatorname{det}\left(A_{G}(\lambda)\right) \\
& =0 .
\end{aligned}
$$

Reasoning in the exactly the same way as above we conclude that $\rho[G]<1$.

Acknowledgements. We wish to thank the anonymous referee for a detailed and careful review of the manuscript, which helped to substantially improve the presentation.

\section{REFERENCES}

[1] P. Lanchster And M. Tismenetsky, The Theory of Matrices, Second Edition with Applications, Academic Press, Orlando, 1985.

[2] J. Stoer And R. Bulirsch, Introduction to Numerical Analysis, Springer-Verlag, New York, 1980. 